\title{
Erbium doped ZBLAN fiber laser operating in the visible - feasibility study
}

\author{
P. Komorowski, ${ }^{* 1,2}$ K. Anders, ${ }^{1}$ U. Zdulska, ${ }^{1}$ and R. Piramidowicz ${ }^{1}$ \\ ${ }^{1}$ Institute of Microelectronics and Optoelectronics, Warsaw University of Technology, \\ Koszykowa 75, Warsaw, 00-662, Poland Poland, \\ ${ }^{2}$ Faculty of Physics, Warsaw University of Technology, Koszykowa 75, Warsaw, 00-662, Poland
}

Received September 22, 2017; accepted September 30, 2017; published September 30, 2017

\begin{abstract}
This work is focused on developing an all-fiber green laser in hybrid geometry, based on a combination of Er:ZBLAN active fiber and silica fiber-based passive components of an optical resonator and deploying Fiber Bragg Gratings (FBGs) as highly selective mirrors for the green spectral range. The scope of work covers a fundamental spectroscopic characterization of Er:ZBLAN samples, determination of key spectroscopic parameters, modelling the lasing properties and lasing experiments in different pumping geometries.
\end{abstract}

Recent years have witnessed continuous development of compact, low-cost, coherent light sources operating within a visible spectral range, driven by numerous applications in optical data processing and storage, photolithography, imaging techniques, medical diagnosis/ therapy, and many others [1]. At present the market is dominated by wide bandgap semiconductor lasers and diode pumped solid state lasers with frequency conversion. It should be noted, however, that less popular fiber lasers, based on low-phonon glasses doped with rare earth ions seem to be an interesting and not fully explored alternative, which could potentially offer higher output powers and better beam quality. In particular, $\mathrm{Er}^{3+}$ doped ZBLAN $\left(53 \mathrm{ZrF}_{4}+20 \mathrm{BaF}_{2}+4 \mathrm{LaF}_{3}+3 \mathrm{AlF}_{3}+20 \mathrm{NaF}\right)$ glass fibers are continuously considered as a promising active medium enabling efficient coherent emission within the green spectral range [2-3]. The structure of erbium energy levels enables efficient excitation using commercially available laser diodes, while a low phonon ZBLAN matrix provides relatively long fluorescence lifetimes of metastable levels. Simultaneously, the fiber geometry offers several advantageous features like easiness of obtaining a single spatial mode operation, excellent heat dissipation and possibility of scaling-up the total gain/output power by simply increasing the length of an active medium while keeping the compact design of the device. This work aims at developing a fiber laser of hybrid design, combining the Er:ZBLAN fiber as an active medium and silica fibers deployed in all remaining passive components of the laser.

The knowledge of spectroscopic properties is essential to predict and model the operation of the investigated laser system. Therefore, a fundamental spectroscopic characterization of Er:ZBLAN bulk samples has been performed, covering the measurements of absorption/ emission characteristics and fluorescence lifetimes, followed by an extraction of main spectroscopic parameters. The visible luminescence characteristics, obtained under direct $(520 \mathrm{~nm})$ and up-converted excitation $(972 \mathrm{~nm})$ are presented in Fig. 1. In the inset the fluorescence decay of ${ }^{4} \mathrm{~S}_{3 / 2}$ level is shown.

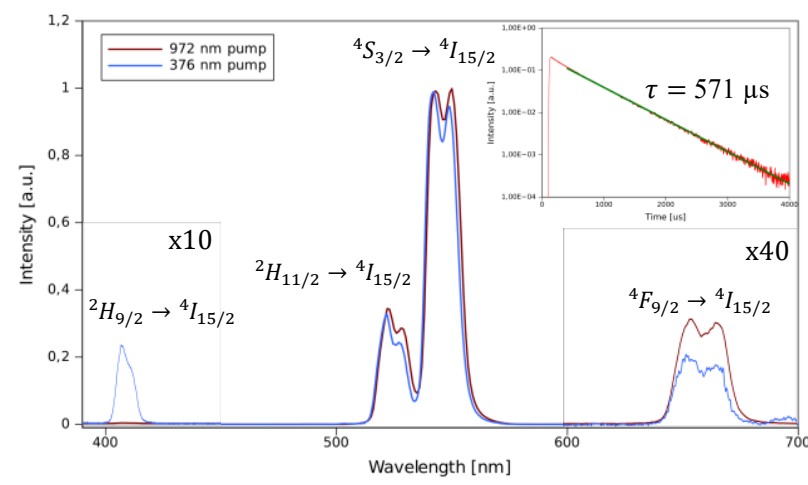

Fig. 1 Emission spectra for two pumping schemes; the inset shows emission decay of ${ }^{4} \mathrm{~S}_{3 / 2}$ level.

Both spectra are dominated by green luminescence, with its maximum at $544 \mathrm{~nm}$, which corresponds to the transition from the ${ }^{4} S_{3 / 2}$ level (coupled thermally with ${ }^{2} \mathrm{H}_{11 / 2}$ ) to the ground state. There are also traces of much weaker emissions in the violet and red part of the spectrum, attributed to transitions from ${ }^{2} \mathrm{H}_{9 / 2}$ and ${ }^{4} \mathrm{~F}_{9 / 2}$, respectively. Apart from the violet line, both spectra have very similar spectroscopic features, irrespective of the excitation mechanism. The fluorescence decays are identical, with a time constant of $571 \mu$ s. This confirms that the excited state absorption mechanism is responsible for up-converted luminescence. The identified excitation and emission processes enabled formulation of the model equations and theoretical analysis of the system.

*E-mail: komorowski.pawel@op.pl 
Two schemes of excitation were analysed, either pumped directly (one-photon pumping) or using upconverted, two-photon pumping (Fig. 2).
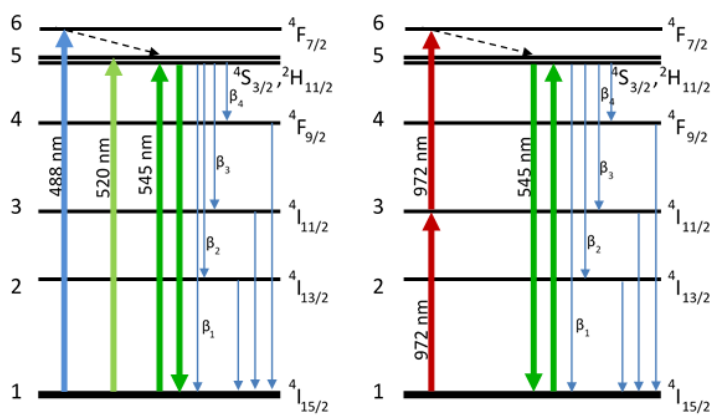

Fig. 2. Direct (left) and up-conversion (right) pumping schemes.

The numerical model is based on two sets of differential equations: rate equations, Eqs. (1), and propagation equations, Eqs. (2), shown below.

$$
\left\{\begin{array}{c}
\frac{d N_{1}}{d t}=-W_{13} * N_{1}+W_{31} * N_{3}+W_{51} * N_{5}-W_{15} * N_{1}+A_{21} * N_{2}+A_{51} * N_{5} \\
\frac{d N_{2}}{d t}=A_{32} * N_{3}-A_{21} * N_{2} \\
\frac{d N_{3}}{d t}=W_{13} * N_{1}-A_{32} * N_{3}-W_{31} * N_{3}-W_{35} * N_{3} \\
\frac{d N_{5}}{d t}=W_{15} * N_{1}-A_{51} * N_{5}-W_{51} * N_{5}+W_{35} * N_{3} \\
N_{1}+N_{2}+N_{3}+N_{5}=1
\end{array}\right.
$$

where: $N_{i}$ - the normalized population of $\mathrm{i}^{\text {th }}$ energy level, $W_{i j}-N_{i} \rightarrow N_{j}$ transition rate, $A_{i j}-N_{i} \rightarrow N_{j}$ relaxation rate.

$$
\begin{aligned}
\frac{d P_{k}}{d z}=\Gamma_{k} * P_{k}(z) * N_{t} * & {\left[N_{\text {upper }}\left(I_{p}, I_{s}\right) * \sigma_{\text {upper-lower }}\right.} \\
& \left.-N_{\text {lower }}\left(I_{p}, I_{S}\right) * \sigma_{\text {lower-upper }}\right]
\end{aligned}
$$

where: $P_{k}$ - the power of $\mathrm{k}^{\text {th }}$ light beam, $\quad \Gamma_{k}-$ the light beam and fiber core overlapping integral, $N_{t}-$ the concentration of the dopant, $\sigma_{i-j}-N_{i} \rightarrow N_{j}$ transition cross-section.

The absorption/emission cross-sections were calculated based on measured spectroscopic characteristics, according to McCumber's theory [4]. The fluorescence lifetimes of individual energy levels were determined from emission decay measurements. The values, used as a set of input parameters for numerical modeling, are summarized in Table 1.

Table 1. Spectroscopic parameters of Er:ZBLAN.

\begin{tabular}{|l|l|l|l|l|}
\hline $\begin{array}{l}\text { Transition cross- } \\
\text { sections }\left[\mathrm{cm}^{2}\right]\end{array}$ & $\sigma_{15}$ & $\sigma_{51}$ & $\sigma_{13}$ & $\sigma_{35}$ \\
\cline { 2 - 4 } & $8.23 \cdot 10^{-21}$ & $7.74 \cdot 10^{-21}$ & $9.41 \cdot 10^{-22}$ & $8.09 \cdot 10^{-22}$ \\
\hline $\begin{array}{l}\text { Fluorescence } \\
\text { lifetimes }[\mathrm{ms}]\end{array}$ & $\tau_{5}$ & $\tau_{3}$ & & \multicolumn{2}{|}{} \\
\cline { 2 - 3 } & 0.571 & 6.67 & &
\end{tabular}

Based on determined spectroscopic parameters, a numerical modelling was performed, with specific attention given to the up-conversion scheme, considered as more attractive. Figure 3 shows the populations of energy levels for up-converted pumping as a function of pumping power.

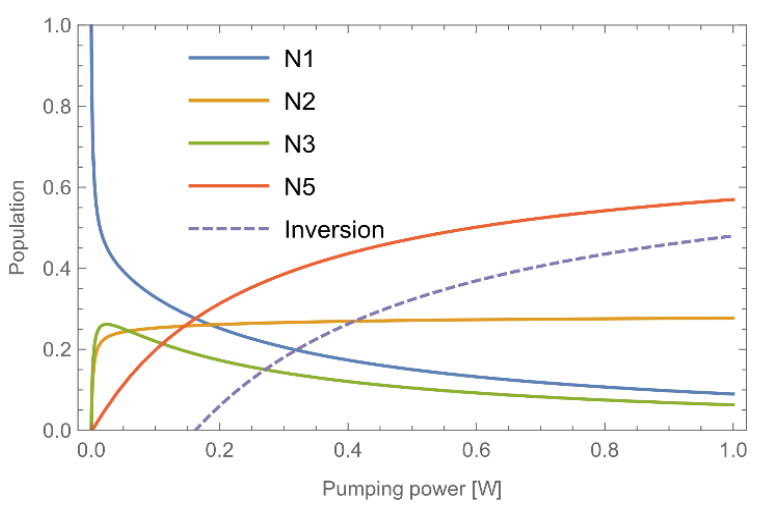

Fig. 3 Populations of energy levels for up-converted pumping.

It is clearly seen that the population inversion (the dashed line in Fig. 3) is obtained for pumping powers higher than $162 \mathrm{~mW}$. A similar dependence, not shown here, was obtained for the direct pumping scheme - in that case the threshold power was $157 \mathrm{~mW}$, which is only few miliwatts lower than for two photon pumping. This shows that the Er:ZBLAN system is a rather unique case, in which up-conversion mechanisms are nearly as efficient as the direct pumping scheme. In Figure 3 one can also see the effect of aggregation of ions in the ${ }^{4} \mathbf{I}_{13 / 2}$ level (denoted as $\mathrm{N}_{2}$ ). This parasitic effect limits the lasing potential of the Er:ZBLAN system both under direct and up-conversion pumping, as $30 \%$ or $27 \%$ of active ions, respectively, are uselessly stored at the ${ }^{4} \mathrm{I}_{13 / 2}$ level.

The distribution of optical power inside the resonator is presented in Fig. 4. The model parameters were chosen intentionally so as to show the influence of the unpumped part of the fiber acting as an absorber (the red part of the figure). This enabled the estimation of an optimal length of the active fiber for a given pumping power.

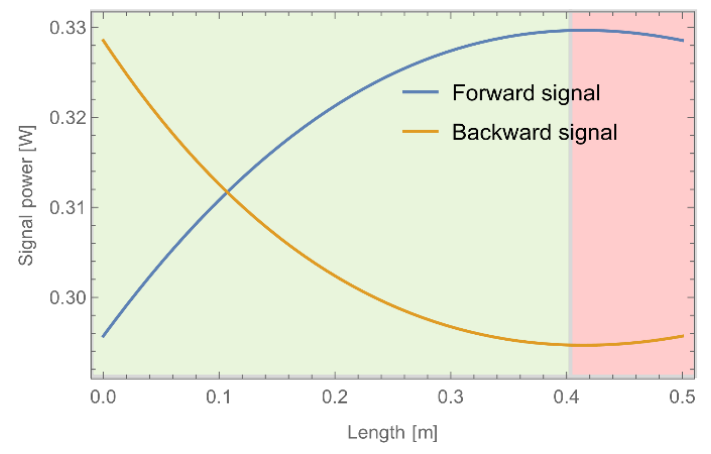

Fig. 4 Distribution of the optical power in the resonator for $500 \mathrm{~mW}$ of up-converted pumping power.

A $300 \mathrm{~mW}$ of launched pumping power finally available during the lasing experiments described below translates to Er:ZBLAN fiber's lengths shorter than $20 \mathrm{~cm}$. 
The general scheme of the investigated Er:ZBLAN fiber laser of hybrid design is shown in Figure 5. Fluorozirconate active fiber was glue spliced with passive silica fibers containing FBG reflectors optimized for the green spectral range. End pumping geometry was implemented with a single pumping source.

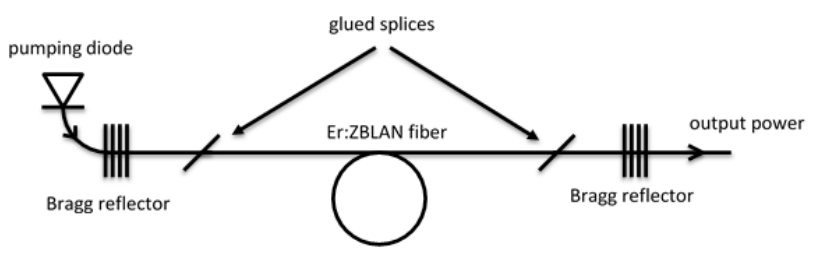

Fig. 5 General scheme of investigated laser.

In the initial experiments a multi-line Ar ion laser was used as a light source, enabling direct excitation of the ${ }^{4} \mathrm{~S}_{3 / 2}$ level. In this case, no laser action has been achieved within the whole range of available pumping powers. The most probable reason was the problem with free-space coupling between pumping laser and active fiber with a core diameter of $5.5 \mu \mathrm{m}$ only.

For experiments with up-converted pumping an all-fiber design has been developed, with a pigtailed, single spatial mode laser diode, operating at $976 \mathrm{~nm}$ used as a pumping source. Again, within all available pumping powers no evidence of laser action has been found. However, despite the lack of evident lasing, near threshold operation has been observed, which is depicted in Figure 6. It should be explained here that although the maximal output power available was ca. $900 \mathrm{~mW}$, the power launched into the active core was significantly smaller (ca. $300 \mathrm{~mW}$ ), due to insertion losses of fusion and glued splices (of the order of $3-4 \mathrm{~dB})$.

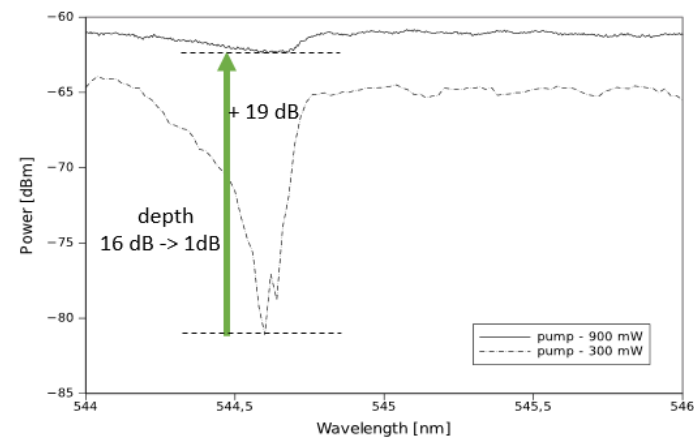

Fig. 6. Emission spectrum dependence on pumping power. The dip centered at $544.8 \mathrm{~nm}$ reflects the shape of FBG, acting as an output coupler.

The results shown in Fig. 6 were obtained for the "best case" configuration, with optimized launching and fiber's length $(15 \mathrm{~cm})$, mitigated insertion losses of glued splices (estimated to be ca. $1.5 \mathrm{~dB}$ ) and highly reflective FBGs (both around 98\%), carefully adjusted mechanically to full overlapping of their spectral characteristics. Under these conditions, an evident decrease of the depth of the dip on the emission spectrum was observed, with increasing pumping power. For a maximal accessible pumping power (estimated to be of order of $300 \mathrm{~mW}$ inside the active core) the emission spectrum was almost completely flattened, which is clearly seen in Fig. 6. This behavior is an evident manifestation of near-threshold operation of the investigated laser system and means that the gain provided by the active medium is nearly compensating all loss mechanisms present in the system and only $1 \mathrm{~dB}$ of gain is missing to get the green lasing. A further increase of pumping power would result in reaching the threshold of laser action, being an ultimate proof of the concept of the hybrid laser.

In conclusions, a design of fiber laser in the hybrid configuration, based on a combination of $\mathrm{Er}^{3+}$ doped ZBLAN active fiber with silica based passive components has been proposed and tested. The laser makes use of a linear resonator, formed by Fiber Bragg Gratings inscribed in the silica fiber connected to both ends of the active ZBLAN fiber. A glue splicing technique has been applied to connect the ZBLAN and silica fibers, differing in physical properties. The investigation of the optical properties of the Er:ZBLAN samples enabled to determine the key spectral parameters of the material, which in turn were used as input data for numerical modeling of the investigated system under direct and upconverted excitation.

Finally, lasing experiments have been performed for different pumping schemes. Although no laser action has been obtained, near-threshold operation has been observed, confirming the potential of the system and clearly suggesting the directions of further work. It should be emphasized here that the proposed design, based on hybrid geometry and FBG mirrors for the green spectral range has no equivalents known from the literature so far.

This work has been supported by the National Science Centre, Poland, agreement number: UMO-2011/ 03/B/ST7/01917. Authors would like to sincerely thank T. Osuch and K. Markowski from the Institute of Electronic Systems, Warsaw University of Technology, for providing the FBG structures.

\section{References}

[1] W.P. Risk, T.R. Gosnell, A.V. Nurmikko, Compact blue-green lasers (Cambridge University Press 2003).

[2] J.Y. Allain, M. Monerie, H. Poignant, Electr. Lett. 28, 111 (1992)

[3] Z . Luo, Q. Ruan, M. Zhong, Y. Cheng, R. Yang, B. Xu, H. Xu, Z. Cai, Opt. Lett. 41, 2258 (2016).

[4] D.E. McCumber, Phys. Rev. 136, 954 (1964). 